\title{
Secondary colon cancer in patients with ulcerative colitis: a systematic review and meta-analysis
}

\author{
Li Zhang ${ }^{1,2}$, Huatian Gan ${ }^{1,3}$ \\ ${ }^{1}$ Department of Gastroenterology and the Center of Inflammatory Bowel Disease, West China Hospital, Sichuan University, Chengdu, China; \\ ${ }^{2}$ Department of Geriatrics, Sichuan Provincial People's Hospital, University of Electronic Science and Technology of China, Chengdu, China; \\ ${ }^{3}$ Lab of Inflammatory Bowel Disease, Clinical institute of Inflammation and Immunology, Frontiers Science Center for Disease-related Molecular \\ Network, West China Hospital, Sichuan University, Chengdu, China \\ Contributions: (I) Conception and design: Both authors; (II) Administrative support: L Zhang; (III) Provision of study materials or patients: Both \\ authors; (IV) Collection and assembly of data: Both authors; (V) Data analysis and interpretation: Both authors; (VI) Manuscript writing: Both \\ authors; (VII) Final approval of manuscript: Both authors. \\ Correspondence to: Huatian Gan. The Center of Inflammatory Bowel Disease, West China Hospital, Sichuan University, 37 Guoxue Lane, Wuhou \\ District, Chengdu, China. Email: ganhuatian@126.com.
}

Background: A meta-analysis was conducted on the incidence of colon cancer in patients with ulcerative colitis (UC). This study aimed to evaluate the correlation between UC and colon cancer, and provide a theoretical guidance for clinical diagnoses and treatments of UC.

Methods: Articles were searched in Chinese database with "ulcerative colitis", "UC", "colon cancer", "colorectal cancer", "incidence", and "meta-analysis" as the search terms. Articles were searched in English database with "ulcerative colitis", "UC", "colon cancer", "incidence rate", and "meta-analysis" as the search terms. Moreover, articles with the topic of "correlation between UC and colon cancer" were screened. The quality of articles was assessed using Rev Man 5.3 software provided by Cochrane system.

Results: Eleven articles were included, most of which were of medium and high quality. Results of metaanalysis showed that 12,216 patients with UC were included in this study, and 110 patients developed colon cancer. There was statistical heterogeneity $\left(\mathrm{Chi}^{2}=103.10, \mathrm{I}^{2}=90 \%, \mathrm{P}<0.00001\right)$. Random-effect model analyses showed that there were no significant differences between colon cancer in patients with UC and those without colon cancer $(\mathrm{Z}=12.44, \mathrm{P}<0.00001)$. A systematic review of articles found that the course and development of colon cancer in patients with UC might affect the occurrence of colon cancer. UC was a risk factor for colorectal cancer.

Discussion: It was found that the course of disease and the occurrence and development of UC might affect the occurrence of colon cancer through a systematic review of articles. UC was one of the risk factors of colorectal cancer.

Keywords: Ulcerative colitis (UC); colitis; meta-analysis

Submitted Oct 27, 2021. Accepted for publication Dec 16, 2021.

doi: 10.21037/jgo-21-800

View this article at: https://dx.doi.org/10.21037/jgo-21-800

\section{Introduction}

Ulcerative colitis (UC) is a chronic nonspecific inflammatory intestinal ulcer in the colon and rectum of unclear etiology with lesions confined to the large bowel mucosa and submucosa (1). Sites of predilection include the large bowel and rectum as well as the colon. Its characteristics are mainly reflected in the formation of ulcers. The disease course is long, easily relapsing, and clinically characterized by diarrhea and abdominal pain as well as mucopurulent stools (2). UC etiology as well as pathogenesis has not been clarified until now, a large number of studies on the disease have been reported, mainly focusing on environmental, 
genetic, infectious as well as immunological factors as well as their interactions (3). Environmental factors have largely followed the degree of development of the country, with studies finding higher rates in developed than developing countries, potentially increasing the incidence of UC; Genetically patients do not have a high incidence of firstdegree relatives and spouses; There are clinical similarities with some infectious bowel diseases, and most scholars believe that bacterial infection may be its precipitating factor (4,5). Immune regulation is abnormal after the disease. Cytokines and inflammatory mediators cause tissue destruction and inflammatory lesions. Massive free radical is formed. Damage to the intestinal mucosa is increased. Now the relatively recognized pathogenesis is that environment factor acts on genetically predisposed persons, the postintestinal flora is involved in initiating the intestinal immune and non-immune systems, the body then develops an immune response and inflammation, and it is clinically presented as UC $(6,7)$. Generally, there are deformation and shortening of the colon, and serious disappearance of the colon pocket and shortening of the intestinal cavity. Cancer may occur in a small number of patients, mostly the undifferentiated $(8,9)$.

Colon cancer is a common gastrointestinal malignancy occurring in the colon. It occurs at the junction of the rectum and sigmoid colon. The incidence is the highest in people in their forties, with men significantly higher than women $(10,11)$. The total incidence of gastrointestinal tumors is the third. The main risk factors for colon cancer are high fat diet and low fiber diet $(12,13)$. The incidence of UC cancer is significantly higher than that of the general population, and genetic factors may also be involved in the development of colon cancer $(14,15)$.

Clinically, the early stage is without any symptoms, and the middle and late stages are characterized by abdominal distension and dyspepsia, after which a change in bowel habit and abdominal pain, mucoid or bloody stools occur $(16,17)$. After tumor ulceration, blood loss, and toxin absorption, symptoms such as anemia, low-grade fever, fatigue, wasting, and lower extremity edema often appear $(18,19)$. Early stage lesions can be treated with endoscopic minimal invasive therapy, and the treatment of intermediate and advanced stage cancer is a comprehensive regimen of surgery based, adjuvant chemotherapy, immunotherapy, traditional Chinese medicine, and other supportive care $(20,21)$. Studies have found that with longer disease duration, the risk of ulcerative colitis lesions developing carcinogenesis will greatly increase, and even there is literature that has included ulcerative colitis as one of the most important several high-risk factors for colon cancer $(22,23)$.

At present, the diagnosis of UC mainly relies on endoscopic and pathological examination, periodic endoscopy and biopsy can detect dysplastic lesions, and the bulge dysplasia detected in the examination of UC patients is heterodysplasia related lesions or masses, with heterodysplasia, the degree of which can be classified as high-grade dysplasia, indeterminate dysplasia and low-grade dysplasia (LGD) $(24,25)$. In recent years, an increasing number of reports on the low-grade dysplasia type have appeared, but a large number of studies involved too few samples, or the specificity of the samples was higher, and the subject of this paper was ulcerative colitis with lowgrade dysplasia (uc-lgd) Patients, the meta-analysis of the incidence of colon cancer, mainly aimed at the references related to the incidence of colon cancer in this type of patients published since the establishment of the library, comprehensive analysis, the occurrence of joint year; to study the correlation between ulcerative colitis and colon cancer, and provide a reference basis for the diagnosis and treatment of digestive diseases such as colon cancer. We present the following article in accordance with the PRISMA reporting checklist (available at https://dx.doi. org/10.21037/jgo-21-800).

\section{Methods}

\section{Article retrieval}

In this study, Chinese and English databases were used to search for "Database of China National Knowledge Network", "Chinese Biomedical Article Database", "Wanfang Database", "Weipu Database", "Baidu Academic", "CompuMath Citation Index", "CMCI", "convolution-based modified clarkson integration", "Medline", "Embase", and "PubMed". A search was performed for all published articles to analyze the incidence of colon cancer in patients with UC-LGD from database construction to June 20, 2021. Compound logic retrieval Boolean logic retrieval method was used to select relevant articles. The articles were searched in Chinese database with "ulcerative colitis", "UC", "colon cancer", "colorectal cancer", "incidence" and, "meta-analysis" as the search terms. English database was searched with "ulcerative colitis", "UC", "colon cancer", "incidence rate", and "metaanalysis" as the search terms. The quality of the articles was assessed using Rev Man 5.3 software provided by Cochrane 
system.

It was suggested to retrieve again after freely combining the various words described above. References that were finally included were determined after multiple searches. Finally, search engines were used to track down the identified articles, and general information of the articles was obtained after careful reading. If perfect information wasn't obtained, it was suggested to contact authors immediately to obtain general information of patients. At the same time, it was advised to contact experts or senior practitioners in the field as soon as possible to get the latest research progress.

\section{Inclusion and exclusion criteria of the included articles}

Included articles should meet all of the following criteria: the type of studies included was a randomized controlled study; patients with UC-LGD who met the clinical criteria were enrolled and were at least 18 years old; patients were followed up with regular clinical examinations and pathological control analyses, and the index comparison was reliable in $95 \%$ confidence interval.

Articles meeting below criteria had to be excluded from this study: subjects of articles were patients with other types of UC; articles which were duplicate published studies, articles without complete original data, and weren't available when the authors were contacted; articles which were case-control studies, cross-sectional studies and case series, relevant conference materials, article reviews, singlecase reports, and lectures and reviews; study data in articles couldn't be extracted completely or couldn't be transformed and used after acquisition; and patients in articles with a history of gastrointestinal tract cases.

Articles were screened by two experts in the field for titles, abstracts, and full texts, and three pre-experiments were conducted before screening. In the end, two experts gave unanimous opinions. If the opinions couldn't be agreed, it was necessary to communicate, discuss, and seek the help of a third party if necessary to reach consistent conclusions.

\section{Data extraction}

Articles were screened by two experts in the field for titles, abstracts, and full texts, and three pre-experiments were conducted before screening. In the end, two experts gave unanimous opinions. If the opinions couldn't be agreed, it was necessary to communicate, discuss, and seek the help of a third party if necessary to reach consistent conclusions. Extracted data in the study included: author, publication time, patients' sample size, time of disease and age, and number of patients with colon cancer.

\section{Bias risk and quality assessments}

Two experts in the industry assessed the risk of bias for included articles. If there were different opinions, consistent conclusions would be drawn through communication and discussion. The study used recommendations from the UK National Institute of Clinical Excellence (NICE) to assess quality. The score range was $0-8 ; 6-8$ indicated high quality research, 3-6 indicated medium quality research, and $0-2$ indicated low quality research. Two researchers were assigned to independently evaluate quality of articles. Consistent conclusions were reached through discussion in case of disagreements. It was necessary to seek the help of a third party to draw conclusions in time if consistent results couldn't be obtained. In the evaluation of risk bias, two researchers made judgments of "a high risk of bias", "a high risk of bias", and "an unclear risk of bias" on each index in the above articles, respectively. It was suggested to use the same method for quality evaluation and give consistent conclusions in case of different opinions.

\section{Statistical methods}

StataSE14.0 software was used for statistical analyses, and the effect sizes were combined. Odds ratio (OR) was used as the effect size index for dichotomous variables, and the standardized mean difference was used as the effect size indicator for continuous variables. Heterogeneity was first tested. If the results of heterogeneity test were $\mathrm{P} \geq 0.1$ and $\mathrm{I}^{2} \leq 50 \%$, it was generally considered that there were no significant differences in heterogeneity between studies and there was certain homogeneity. Therefore, fixedeffect models were used for meta-analysis. When the heterogeneity test result was $\mathrm{P}<0.1, \mathrm{I}^{2}>50 \%$, it was generally considered that the studies weren't homogenous and had significant heterogeneity. Random effect models were used for meta-analysis. In the calculation, the comprehensive influence that led to heterogeneity and results of the study was excluded, so that the reliability of the results was more guaranteed. The bias risk assessment diagram of Rev Man 5.3 was used to evaluate the risk bias of included articles, and $95 \%$ confidence interval was required for the effect of each indicator. 


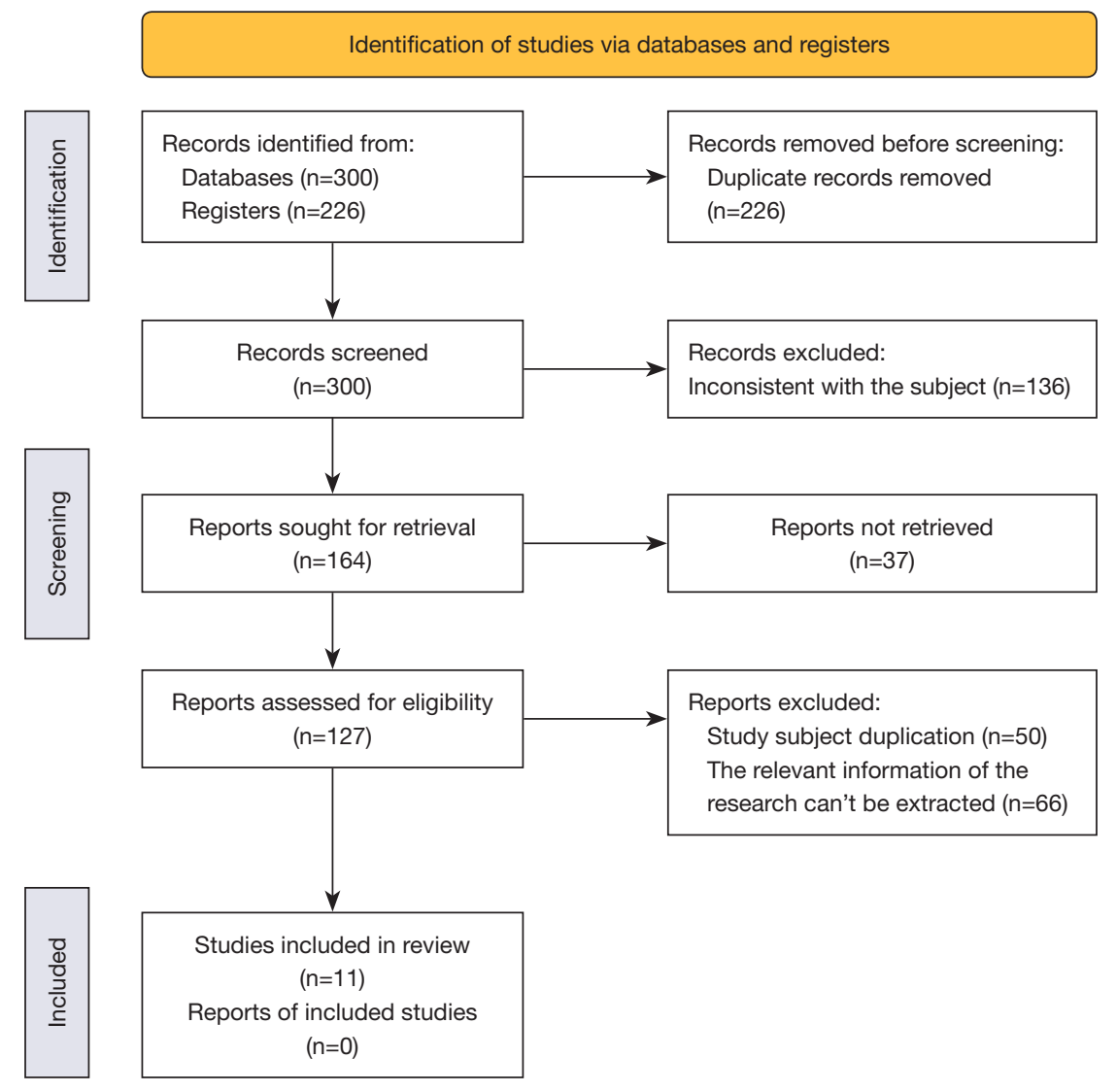

Figure 1 Retrieval flow chart.

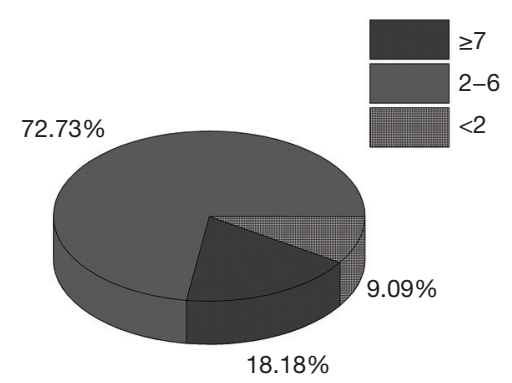

Figure 2 Quality assessment results of NICE study.

\section{Results}

\section{Search results and basic information of included documents}

Three hundred articles were obtained from Chinese database, and 226 from English database. A total of 526 articles were obtained using the combination strategy. The remaining 300 articles were obtained by excluding 226 duplicates. One hundred thirty six articles were screened after reading abstracts and titles, and the remaining 164 articles were screened. One hundred fifty three articles were excluded and 11 articles were finally included for meta-analysis after a thorough reading of full texts. The article retrieval flow chart was shown in Figure 1. The main reasons for article exclusion were as follows: included subjects of 37 articles with diseases of other systems and organs or serious mental diseases; study objects of 20 articles were repeated; 30 articles related to the study couldn't be obtained completely even after contacting the authors; and 66 articles lacking relevant study results and original data. Figure 2 showed the quality evaluation results on account of the recommendation of NICO. It could be shown from the figure that the quality assessment of articles. There were 2 articles with $\geq 7$ points, accounting for $18.18 \%, 8$ articles with 2-6 points, accounting for $72.73 \%$, and 1 article with $<2$ points, accounting for $9.09 \%$.

Eleven articles met the inclusion criterion. According to the statistics of the study objects in articles, the sample size 
Table 1 Basic features of the included literature

\begin{tabular}{|c|c|c|c|c|c|}
\hline The first author & $\begin{array}{c}\text { Year of } \\
\text { publication }\end{array}$ & Age (years old) & $\begin{array}{c}\text { The total number } \\
\text { of patients }\end{array}$ & $\begin{array}{l}\text { Number of patients } \\
\text { with colon cancer }\end{array}$ & $\begin{array}{c}\text { Course of disease } \\
\text { (years) }\end{array}$ \\
\hline Shivakumar (12) & 2013 & $46(36-54.5)$ & 29 & 1 & $7.5-14.5$ \\
\hline Desai (13) & 2015 & $44.5 \pm 14.6$ & 430 & 12 & 6 [1-39] \\
\hline Kim (14) & 2009 & $37.9 \pm 17.1$ & 7061 & 26 & - \\
\hline Gilat (17) & 1988 & $36.6 \pm 16.5$ & 1035 & 26 & - \\
\hline Kiesslich (18) & 2003 & $42.2 \pm 12.1$ & 165 & 8 & $11.2 \pm 7.3$ \\
\hline Kekilli (19) & 2010 & $35.9 \pm 13.5$ & 275 & 3 & $15.1 \pm 5.2$ \\
\hline
\end{tabular}

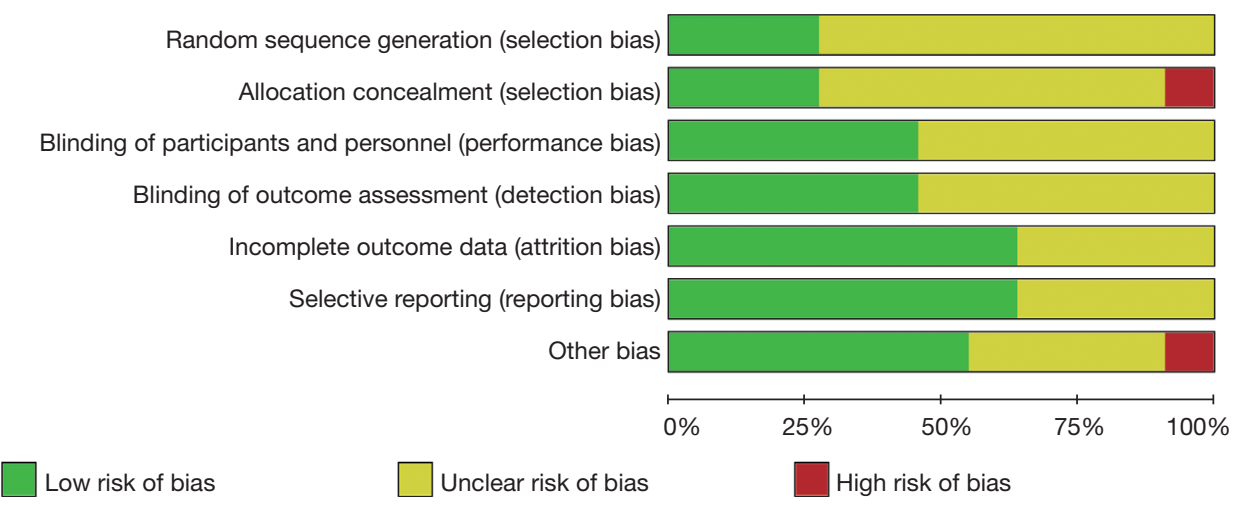

Figure 3 Evaluation results of article risk bias.

was 12,216. Most of the included articles were small sample studies. Statistical results showed that the sample size of articles was small. General information of patients described in 11 articles included age, duration of disease, occurrence of colon cancer, and others. Table 1 below was the general information of the study objects in the included articles which covered from 1988 to 2015.

\section{Results of references risk bias evaluations}

Figure 3 and Figure 4 showed the results of multiple risk bias evaluations of references drawn by Review Manager software. For each methodological feature included in the articles, it was suggested to input the evaluation results into software to generate bias risk assessment map. Random sequence generation (selection bias), allocation concealment (selection bias), the results of evaluation of blinded (bias), and the result of incomplete result data (follow-up bias), selective report (report bias) significantly at a low risk of bias. Participants and researchers blinded (bias) and a low risk of bias assessments of other bias were about $50 \%$. The risk bias of other articles was significantly a low risk of bias except articles of Kamiya [2015] and Gilat [1988].

\section{Situation of UC complicated with colon cancer}

11 articles analyzed the overall risks of developing colorectal cancer in patients with UC. Figure 5 showed the 


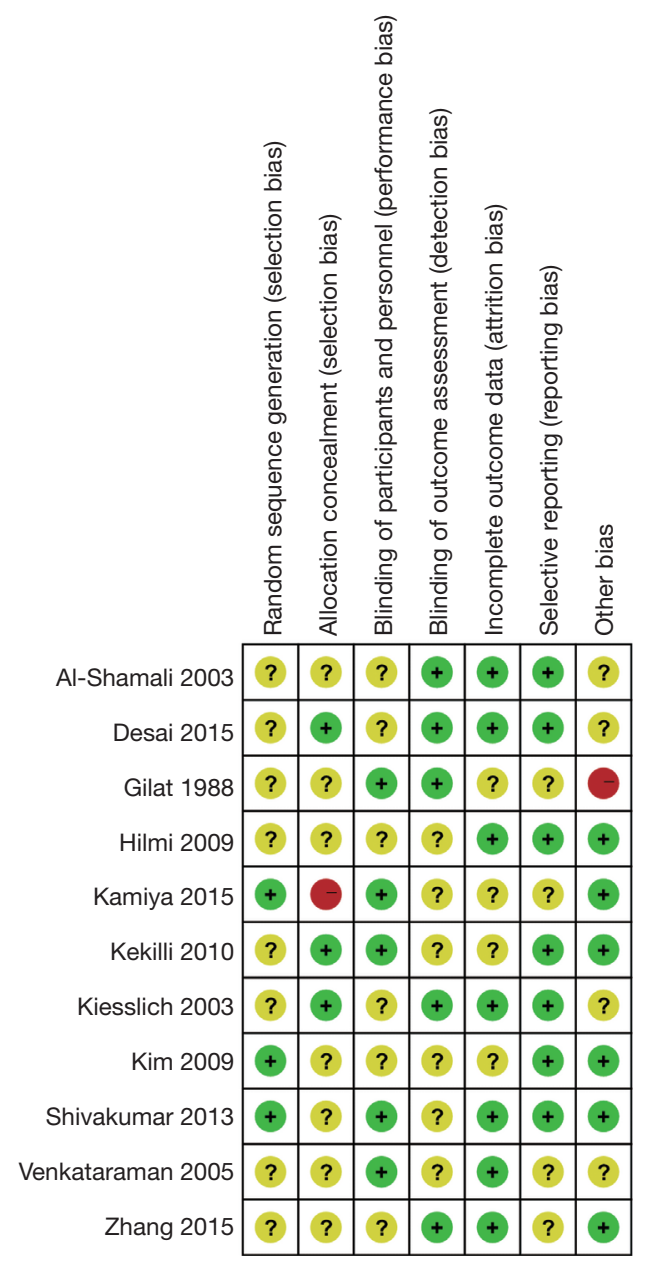

Figure 4 Evaluation results of multiple risk bias corresponding to multiple studies in articles.

comparative meta-analysis results of included patients with UC who developed colorectal cancer and those who didn't. It could be shown that the results of Gilat [1988], Kamiya [2015], and Kim [2009] all accounted for a high percentage $(11.6 \%)$ in the final combined results, followed by the results of Desai [2015] (11.2\%). In addition, the horizontal line of the $95 \%$ confidence interval for most studies was to the left of the invalid vertical line. Among the 11 article included, 12,216 patients with UC were included, including 110 patients who developed colon cancer. There was statistical heterogeneity $\left(\mathrm{Chi}^{2}=103.10, \mathrm{I}^{2}=90 \%, \mathrm{P}<0.00001\right)$. The combined effect size or diamond block didn't cross with invalid vertical line, OR value was 0.01 , and $95 \%$ confidence interval was 0.01 and 0.03 . Random-effect model analyses showed that there were no significant differences between patients with UC who developed colon cancer and those without colon cancer $(\mathrm{Z}=12.44, \mathrm{P}<0.00001)$.

Figure 6 showed publication bias. In the 6 studies, the incidence of UC related colon cancer was analyzed, and OR value was set as the horizontal plane and the standard error as the vertical plane to draw a funnel plot, as shown in Figure 6 below. The circles in the included articles were concentrated in the top region with high research accuracy, and were distributed on both sides of the invalid midline, which was uniform and roughly symmetrical. Therefore, there was no significant publication bias in the included studies.

\section{Discussion}

With the development of global economy, there are more and more UC patients at home and abroad, and research reports on UC also increase. If UC is left untreated, it will have a high chance of developing colon cancer $(26,27)$. Currently, despite great advances in the treatment of colon cancer, the 5-year survival rate for advanced colon cancer has not changed much over the years, and therefore, the meaningful healing of colon cancer prevention is important. According to the multistage theory of the carcinogenesis process, the occurrence of colon cancer also goes through 3 stages: initiation, promotion, and progression, and morphologically, it is manifested as normal mucosa $\rightarrow$ hyperplasia $\rightarrow$ adenoma formation $\rightarrow$ adenoma carcinogenesis $\rightarrow$ invasive metastasis, such as when the carcinogenesis of familial adenomatous polyposis is used as a model, and the natural history of colon cancer can be as long as $10-35$ years, This represents a highly advantageous opportunity for colon cancer prevention, which may be approached using the following preventive strategies that, depending on the various stages of the colon cancer natural history, eliminate or reduce exposure of the large bowel mucosa to carcinogenic agents before tumorigenesis, inhibit or block the carcinogenic process in epithelial cells, and thus prevent neoplasia. These include dietary interventions, Chemoprevention and treatment of precancerous lesions. Meta-analysis was adopted in this study. Among the 11 articles included, 10 were randomized controlled grouping and only 1 was retrospective analysis, which brought bias to the study. But in general, the effect on the results of this study was small. Studies on a single sample could be unstable. Using meta-analysis to quantitatively synthesize each included article in the study couldn't only avoid differences between studies that might be caused by sampling from different population, but also assigned 


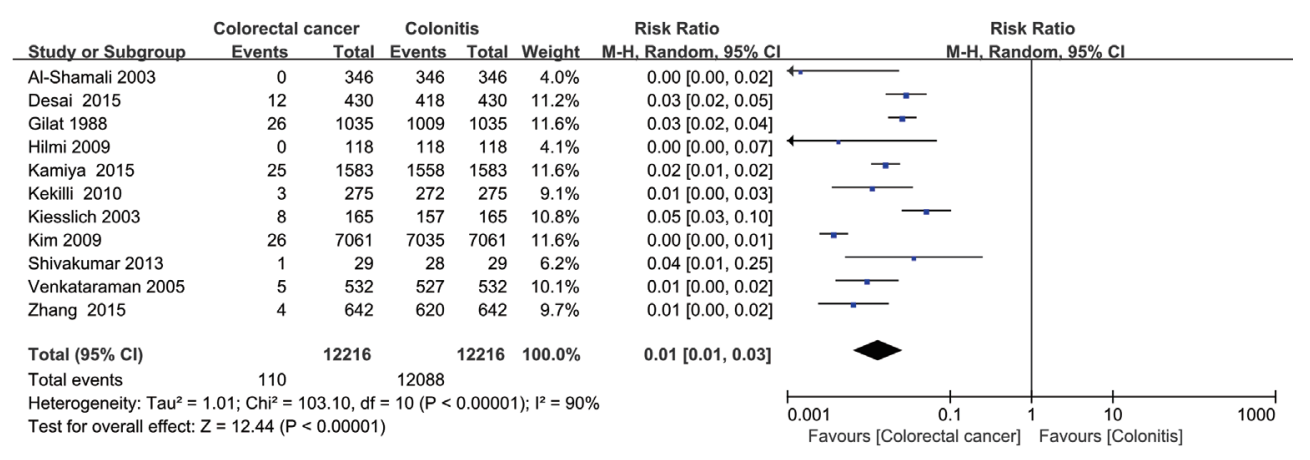

Figure 5 Forest map of colon cancer combined annual incidence.

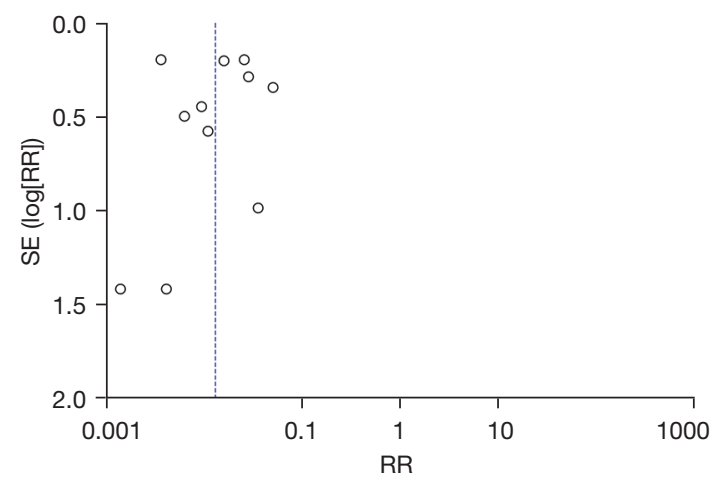

Figure 6 Publication bias funnel plot of comorbidities of colon cancer.

different weights to the results according to the sample size of each study, so as to increase the sample size and improve the credibility of the conclusion. The quality of metaanalysis mainly depended on the authenticity and integrity of the articles. Due to the objective influence of included articles, the number of included articles in this study was limited. Follow-up studies should increase the sample size to prevent bias in analyses.

In this study, the recommendations of NICO were used to evaluate the quality of the included articles, and the scoring criteria were strictly followed. It was concluded that there were 2 articles with scores of $\geq 7$ points, accounting for $18.18 \%, 8$ articles with scores of $2-6$ points, accounting for $72.73 \%$, and 1 article with scores of $<2$ points, accounting for $9.09 \%$. According to the evaluation results, articles included in this study had a good credibility. A total of 11 articles were included in this meta-analysis. The incidence of colon cancer in patients with UC was analyzed to evaluate the association between $\mathrm{UC}$ and colon cancer. In a meta-analysis of UC-LGD colon cancer incidence, a total of 11 articles analyzed overall risks of developing colorectal cancer in patients with UC. A total of 12,216 patients with UC were included, including 110 patients who developed colon cancer. There was statistical heterogeneity $\left(\mathrm{Chi}^{2}=103.10, \mathrm{I}^{2}=90 \%, \mathrm{P}<0.00001\right)$. Random-effect model analyses showed that there were no significant differences between patients with $\mathrm{UC}$ who developed colon cancer and those without colon cancer $(\mathrm{Z}=12.44, \mathrm{P}<0.00001)$. It indicated that the course of disease and the occurrence and development of UC might affect the occurrence of colon cancer. UC was one of the risk factors of colorectal cancer. The circles included in the study were concentrated in the top region with high precision, and were distributed on both sides of the invalid midline, which was uniform and roughly symmetrical. The results showed that there was no significant publication bias in the included studies. In general, the quality of the articles included in the metaanalysis of this study was medium and above.

\section{Conclusions}

In this study, 11 articles were included in the Boolean logic retrieval method of compound logic retrieval. A metaanalysis was conducted to evaluate the incidence of colon cancer in patients with UC to investigate the correlation between US and colon cancer. Results showed that the course of disease and the occurrence and development of UC might affect the occurrence of colon cancer. UC was one of the risk factors of colorectal cancer. Limitations of this study lied in the concentrated distribution of patient samples and a small number of subjects. Other diseases might also affect the incidence of colon cancer. If the influence wasn't removed, the final analysis conclusion would have a certain degree of deviation, and the final conclusion might also be affected by subjective factors of 
doctors and different body differences and drug responses. In conclusion, this meta-analysis could provide a reference for the prevention of the development of colon cancer in patients with UC.

\section{Acknowledgments}

Funding: The present work was supported by the National Natural Science Foundation of China (No. 81470826), the Science Foundation from Science \& Technology Department of Sichuan Province (No. 2019YFS0262; No. 2020YFS0410; No. 22ZDYF1691; No. 2018FZ0062) and 1.3.5 project for disciplines of excellence, West China Hospital, Sichuan University (No. ZYGD18023).

\section{Footnote}

Reporting Checklist: The authors have completed the PRISMA reporting checklist. Available at https://dx.doi. org/10.21037/jgo-21-800

Conflicts of Interest: Both authors have completed the ICMJE uniform disclosure form (available at https://dx.doi. org/10.21037/jgo-21-800). The authors have no conflicts of interest to declare.

Ethical Statement: The authors are accountable for all aspects of the work in ensuring that questions related to the accuracy or integrity of any part of the work are appropriately investigated and resolved.

Open Access Statement: This is an Open Access article distributed in accordance with the Creative Commons Attribution-NonCommercial-NoDerivs 4.0 International License (CC BY-NC-ND 4.0), which permits the noncommercial replication and distribution of the article with the strict proviso that no changes or edits are made and the original work is properly cited (including links to both the formal publication through the relevant DOI and the license). See: https://creativecommons.org/licenses/by-nc-nd/4.0/.

\section{References}

1. Kucharzik T, Koletzko S, Kannengiesser K, et al. Ulcerative Colitis-Diagnostic and Therapeutic Algorithms. Dtsch Arztebl Int 2020;117:564-74.

2. Blanchaert C, Strubbe B, Peeters H. Fecal microbiota transplantation in ulcerative colitis. Acta Gastroenterol
Belg 2019;82:519-28.

3. Yang L, Bian Y, Li Z, et al. Identification of potential biomarkers and pathways in ulcerative colitis with combined public mRNA and miRNA expression microarray data analysis. J Gastrointest Oncol 2019;10:847-58.

4. Singh S, Feuerstein JD, Binion DG, et al. AGA Technical Review on the Management of Mild-to-Moderate Ulcerative Colitis. Gastroenterology 2019;156:769-808.e29.

5. Wen J, Min X, Shen M, et al. ACLY facilitates colon cancer cell metastasis by CTNNB1. J Exp Clin Cancer Res 2019;38:401.

6. Jun X, Ning C, Yang S, et al. Alteration of Fungal Microbiota After 5-ASA Treatment in UC Patients. Inflamm Bowel Dis 2020;26:380-90.

7. Gelibter AJ, Caponnetto S, Urbano F, et al. Adjuvant chemotherapy in resected colon cancer: When, how and how long? Surg Oncol 2019;30:100-7.

8. Eaton JE, Smyrk TC, Imam M, et al. The fate of indefinite and low-grade dysplasia in ulcerative colitis and primary sclerosing cholangitis colitis before and after liver transplantation. Aliment Pharmacol Ther 2013;38:977-87.

9. Goldstone R, Itzkowitz S, Harpaz N, et al. Progression of low-grade dysplasia in ulcerative colitis: effect of colonic location. Gastrointest Endosc 2011;74:1087-93.

10. Dong B, Chai M, Chen H, et al. Screening and verifying key genes with poor prognosis in colon cancer through bioinformatics analysis. Transl Cancer Res 2020;9:6720-32.

11. Zisman TL, Bronner MP, Rulyak S, et al. Prospective study of the progression of low-grade dysplasia in ulcerative colitis using current cancer surveillance guidelines. Inflamm Bowel Dis 2012;18:2240-6.

12. Shivakumar BM, Lakshmankumar B, Rao L, et al. Colorectal neoplasia in long-standing ulcerative colitis - a prospective study from a low-prevalence area. Colorectal Dis 2013;15:e462-8.

13. Desai D, Shah S, Deshmukh A, et al. Colorectal cancers in ulcerative colitis from a low-prevalence area for colon cancer. World J Gastroenterol 2015;21:3644-9.

14. Kim BJ, Yang SK, Kim JS, et al. Trends of ulcerative colitis-associated colorectal cancer in Korea: A KASID study. J Gastroenterol Hepatol 2009;24:667-71.

15. Hilmi I, Singh R, Ganesananthan S, et al. Demography and clinical course of ulcerative colitis in a multiracial Asian population: a nationwide study from Malaysia. J Dig Dis 2009;10:15-20.

16. Zhang Q, Sha S, Xu B, et al. Prevalence of colorectal 
cancer in patients with ulcerative colitis: A retrospective, monocenter study in China. J Cancer Res Ther 2015;11:899-903.

17. Gilat T, Fireman Z, Grossman A, et al. Colorectal cancer in patients with ulcerative colitis. A population study in central Israel. Gastroenterology 1988;94:870-7.

18. Kiesslich R, Fritsch J, Holtmann M, et al. Methylene blue-aided chromoendoscopy for the detection of intraepithelial neoplasia and colon cancer in ulcerative colitis. Gastroenterology 2003;124:880-8.

19. Kekilli M, Dagli U, Kalkan IH, et al. Low incidence of colorectal dysplasia and cancer among patients with ulcerative colitis: a Turkish referral centre study. Scand J Gastroenterol 2010;45:434-9.

20. Kamiya T, Ando T, Watanabe O, et al. Suitability of surveillance colonoscopy for patients with ulcerative colitis to detect colorectal cancer: current guidelines miss some early-stage cases. Nagoya J Med Sci 2015;77:237-44.

21. Venkataraman S, Mohan V, Ramakrishna BS, et al. Risk of colorectal cancer in ulcerative colitis in India. J Gastroenterol Hepatol 2005;20:705-9.

22. Al-Shamali MA, Kalaoui M, Patty I, et al. Ulcerative colitis in Kuwait: a review of 90 cases. Digestion 2003;67:218-24.

23. Eaden J, Abrams K, Ekbom A, et al. Colorectal cancer prevention in ulcerative colitis: a case-control study. Aliment Pharmacol Ther 2000;14:145-53.

24. Rutter M, Saunders B, Wilkinson K, et al. Severity of inflammation is a risk factor for colorectal neoplasia in ulcerative colitis. Gastroenterology 2004;126:451-9.

25. Moody GA, Jayanthi V, Probert CS, et al. Long-term therapy with sulphasalazine protects against colorectal cancer in ulcerative colitis: a retrospective study of colorectal cancer risk and compliance with treatment in Leicestershire. Eur J Gastroenterol Hepatol 1996;8:1179-83.

26. Lashner BA, Provencher KS, Seidner DL, et al. The effect of folic acid supplementation on the risk for cancer or dysplasia in ulcerative colitis. Gastroenterology 1997;112:29-32.

27. Lynch DA, Lobo AJ, Sobala GM, et al. Failure of colonoscopic surveillance in ulcerative colitis. Gut 1993;34:1075-80.

(English Language Editor: L. Huleatt)
Cite this article as: Zhang L, Gan H. Secondary colon cancer in patients with ulcerative colitis: a systematic review and metaanalysis. J Gastrointest Oncol 2021;12(6):2882-2890. doi: 10.21037/jgo-21-800 